\title{
Joint TOA and DOA Estimation for UWB Systems with Antenna Array via Doubled Frequency Sample Points and Extended Number of Clusters
}

\author{
Liangliang Gong $\mathbb{D}$, Yang Hu, Junyao Zhang, and Gaofeng Zhao \\ NARI Group Corporation (State Grid Electric Power Research Institute), Nanjing 211000, China \\ Correspondence should be addressed to Liangliang Gong; gongliangliangnr@163.com
}

Received 12 April 2021; Accepted 29 May 2021; Published 7 June 2021

Academic Editor: Junpeng Shi

Copyright (c) 2021 Liangliang Gong et al. This is an open access article distributed under the Creative Commons Attribution License, which permits unrestricted use, distribution, and reproduction in any medium, provided the original work is properly cited.

\begin{abstract}
The ultra-wideband (UWB) system, which transmits information using nanosecond or even sub-nanosecond pulses, has been widely applied in precise positioning. In this paper, we investigate the problem of the time of arrival (TOA) estimation and the direction of arrival (DOA) estimation in the UWB systems with antenna array and propose a joint TOA and DOA estimation algorithm with doubled frequency sample points and extended number of clusters. Specifically, the proposed algorithm uses two antennas to receive impinging signals and utilizes the conjugate symmetry characteristic of the delay matrices to extend the sample points as well as the number of clusters. Moreover, in order to obtain TOA estimates with low computational complexity, the proposed algorithm transforms the two-dimensional (2D) spectral search to one-dimensional (1D) searches. The DOA estimates can then be achieved by using the TOA estimation results and the geometric information. Simulation results are given to testify the performance of the proposed algorithm.
\end{abstract}

\section{Introduction}

The ultra-wideband (UWB) technique is a kind of wireless communication technology which uses nanosecond or even sub-nanosecond pulses as carrier to transmit information. Benefiting from the high transmission rate, low power consumption, and the anti-multipath characteristics, the UWB system has been widely applied in various fields such as radar, imaging, and positioning [1-3]. Due to the extremely narrow pulse, the positioning precision of the UWB system can reach centimeter level or even millimeter level. Even under complex multipath conditions such as indoor environment, the UWB system can still achieve accurate positioning due to its strong anti-multipath and penetration ability [4-6]. At present, IEEE 802.15.4a standard has taken UWB as the preferred technology for positioning application [7].

One of the basic problems in the UWB positioning system is the time of arrival (TOA) estimation. TOA estimation algorithms can be divided into two categories: one is the traditional algorithms based on the time domain, and the other is the high-resolution algorithms based on the frequency domain. The former mainly includes the coherent detection method using pulse template matched filter [8] and the incoherent TOA estimation algorithm based on threshold or energy detection [9-11]. The coherent algorithm based on matched filter can obtain TOA estimates with high accuracy but at the same time with high sampling rate, complex receiver structure, and expensive equipment cost. The incoherent TOA estimation algorithm has the advantages of low sampling rate, fast convergence speed, and low hardware resource occupation rate. However, the low sampling rate leads to low time resolution, which reduces the accuracy of the TOA estimation. The traditional time-domain-based algorithms obtain TOA estimates by estimating the arrival time of the direct path (DP) component in the received signal. However, due to the multipath effect and the nonline-of-sight (NLOS) condition, DP may not be the 
strongest path; thus the algorithm resolution declines. Therefore, super-resolution estimation algorithms based on frequency-domain processing are proposed [12-18]. These algorithms, such as the multiple signal classification (MUSIC) algorithm [12, 13], the propagator method (PM) [14], the estimation of signal parameters via rotational invariance techniques (ESPRIT) algorithm $[15,16]$, and the matrix pencil algorithm [17, 18], model the channel impulse response in frequency domain and realize the TOA estimation using the orthogonality between the signal subspace and the noise subspace, which can achieve high estimation resolution.

Researches on DOA estimation in UWB systems were also done. Since the UWB system has high time resolution, some joint TOA and DOA estimation algorithms were proposed. The matrix pencil algorithm was applied to the joint estimation in [19]. In [20], the rough TOA estimates were obtained via energy estimation and the minimum distance criterion, the accurate estimates were then achieved by a low-complexity and high-resolution method based on the signal power delay spectrum, and the DOA estimates were finally obtained by the minimum-variance unbiased estimation using the TOA estimation results. Besides, the electromagnetic vector sensor (EMVS) with multiple-input multiple-output (MIMO) radar [21] also has the potential to be exploited in the UWB systems.

In this paper, we investigate the problem of joint TOA and DOA estimation in UWB systems and propose a computationally efficient algorithm. Specifically, the proposed algorithm uses two antennas to receive impinging signals and utilizes the conjugate symmetry characteristic of the delay matrices to double the equivalent frequency sample points and extend the number of clusters. Moreover, to obtain TOA estimates with low computational complexity, the proposed algorithm transforms the two-dimensional (2D) spectral search to one-dimensional (1D) searches. The DOA estimates can then be achieved by using the TOA estimation results and the geometric information. The main contributions of this paper are summarized as follows:

(1) We propose an effective algorithm for UWB systems to jointly obtain the DOA and TOA estimation results.

(2) We transform the time-consuming 2D spectral search to twice $1 \mathrm{D}$ searches in order to release the computational burden.

(3) The proposed algorithm can obtain high estimation accuracy due to the doubled sample points and the extended number of clusters.

The rest of this paper is organized as follows: Section 2 introduces the data model of the UWB system. The proposed joint TOA and DOA estimation algorithm is derived in Section 3. Section 4 then analyses the estimation performance of the proposed algorithm and Section 5 gives the simulation results to testify the algorithm effectiveness. Finally, Section 6 concludes this paper.

Notation 1. In this paper, we use upper (lower) bold characters to represent matrices (vectors). $(\cdot)^{*},(\cdot)^{T}$, and $(\cdot)^{H}$, respectively, denote the operation of conjugate, transpose, and conjugate transpose. * denotes the convolution operation, and $\otimes$ denotes the Kronecker product. $\mathbf{A}(:, m: n)$ generates a new matrix consisted by the $m$-th to the $n$-th columns of the matrix A. $E\{\cdot\}$ represents the expectation operation. $\mathbf{I}_{M}$ stands for an $M \times M$ identity matrix.

\section{Data Model}

2.1. Transmit Signals of UWB Systems. In this paper, we utilize the second derivation of the Gaussian pulse as the UWB transmit signal and modulate it with the direct sequence binary phase shift keying (DS-BPSK). The transmit signal of the UWB system can then be expressed as [22]

$$
s(t)=\sum_{\gamma=-\infty}^{+\infty} \sum_{n=0}^{N_{c}-1} b_{\gamma} c_{n} p\left(t-\gamma T_{s}-n T_{c}\right),
$$

where $b_{\gamma} \in\{-1,+1\}$ is the modulated binary data symbol sequence, $c_{n} \in\{-1,+1\}$ is the pseudorandom sequence used to realize the multiple access communication, $T_{c}$ denotes the repeat period of the pulse, $T_{s}$ represents the period of the binary data symbol, and $N_{c}$ is the pulse repetition times of a single binary data symbol. The term $p(t)$ is the second derivation of the Gaussian pulse and can be further expressed as

$$
p(t)=e^{-\left(2 \pi t^{2} / \Gamma^{2}\right)}\left(1-\left(4 \pi t^{2} / \Gamma^{2}\right)\right),
$$

where $\Gamma$ is the pulse forming factor related to the pulse width.

2.2. Channel Model of UWB Systems. According to the Saleh-Valenzuela (SV) model [23], we consider that the transmit signal produces multiple multipath components after passing through the channel, and these multipath components arrive at the receiver in the form of clusters. Specifically, we assume there are $K$ clusters and $L$ multipath per cluster in the UWB channel, then the channel impulse response of the $k$ th cluster is given by

$$
h^{(k)}(t)=\sum_{l=1}^{L} \alpha_{l}^{(k)} e^{j \theta_{l}^{(k)}} \delta\left(t-\tau_{l}^{(k)}\right),
$$

where $\alpha_{l}^{(k)}$ is the channel fading factor of the $l$ th path in the $k$ th cluster, which obeys the Rayleigh distribution. $\theta_{l}^{(k)}$ distributes uniformly in the range $[0,2 \pi], \delta(\cdot)$ is the Dirac function, and $\tau_{l}^{(k)}$ is the channel delay of the $l$ th path in the $k$ th cluster. Normally, the change rate of the channel is slow compared with the pulse speed of the transmit signal; hence we have $\tau_{l}^{(k)}=\tau_{l}$. Define $\beta_{l}^{(k)}=\alpha_{l}^{(k)} e^{j \theta_{l}^{(k)}}$ to denote the random complex fading amplitude, then (3) can be rewritten as

$$
h^{(k)}(t)=\sum_{l=1}^{L} \beta_{l}^{(k)} \delta\left(t-\tau_{l}\right) .
$$

2.3. Received Signals of UWB Systems. According to the basic theory of the digital signal processing, the received signal of the $k$ th cluster in the time domain can be expressed as [23] 


$$
\begin{aligned}
y^{(k)}(t) & =s(t) * h^{(k)}(t)+w^{(k)}(t) \\
& =\sum_{l=1}^{L} \sum_{\gamma=-\infty}^{+\infty} \sum_{n=1}^{N_{c}-1} \beta_{l}^{(k)} b_{\gamma} c_{n} p\left(t-\gamma T_{s}-n T_{c}-\tau_{l}\right)+w^{(k)}(t)
\end{aligned}
$$

where $w^{(k)}(t)$ is the additive Gaussian white noise of the $k$ th cluster. Transform (5) into frequency domain, i.e.,

$$
\begin{aligned}
Y^{(k)}(\omega) & =S(\omega) H^{(k)}(\omega)+W^{(k)}(\omega) \\
& =\sum_{l=1}^{L} \beta_{l}^{(k)} S(\omega) e^{-j \omega \tau_{l}}+W^{(k)}(\omega),
\end{aligned}
$$

where $Y^{(k)}(\omega), S(\omega), H^{(k)}(\omega)$, and $W^{(k)}(\omega)$, respectively, represent the Fourier transformation of $y^{(k)}(t), s(t), h^{(k)}(t)$, and $w^{(k)}(t)$. Subsequently, by sampling $N(N>L)$ points with interval $\Delta \omega=(2 \pi / N)$ in the frequency domain, we can obtain the measurement data vector as [23]

$$
\mathbf{y}_{k}=\mathbf{S E}(\tau) \boldsymbol{\beta}_{k}+\mathbf{w}_{k},
$$

where vector $\mathbf{y}_{k}=\left[Y^{(k)}\left(\omega_{0}\right), \ldots, Y^{(k)}\left(\omega_{N-1}\right)\right]^{T}$ with $\omega_{n}=$ $n \Delta \omega(n=0,1, \ldots, N-1) . \mathbf{S}=\operatorname{diag}\left\{S\left(\omega_{0}\right), \ldots, S\left(\omega_{N-1}\right)\right\}$ is an $N \times N$ diagonal matrix. $\mathbf{E}(\tau)=\left[\mathbf{e}\left(\tau_{1}\right), \mathbf{e}\left(\tau_{2}\right), \ldots,, \mathbf{e}\left(\tau_{L}\right)\right]$ is the delay matrix which contains the information of the signal multipath delay, where $\mathbf{e}\left(\tau_{i}\right)=\left[1, e^{-j \Delta \omega \tau_{i}}, \ldots\right.$, $\left.e^{-j(N-1) \Delta \omega \tau_{i}}\right]^{T}$. Moreover, $\beta_{k}=\left[\beta_{1}^{(k)}, \beta_{2}^{(k)}, \ldots, \beta_{L}^{(k)}\right]^{T}$ contains the complex channel fading factor of the $k$ th cluster and $\mathbf{w}_{k}=\left[W^{(k)}\left(\omega_{0}\right), W^{(k)}\left(\omega_{1}\right), \ldots, W^{(k)}\left(\omega_{N-1}\right)\right]^{T}$ is the observe vector of the noise.

In this paper, we use two array antennas to receive the impinging signals. As shown in Figure 1, we assume there are $L$ far-field signals from direction $\left\{\theta_{1}, \theta_{2}, \ldots, \theta_{L}\right\}$. The TOA of the two antennas is $\tau=\left[\tau_{1}, \tau_{2}, \ldots, \tau_{L}\right]$ and $\varsigma=\left[\varsigma_{1}, \varsigma_{2}, \ldots, \varsigma_{L}\right]$, respectively. The distance between the two antennas is $d$, and $c$ denotes the light speed. According to (7), the received signal of the two antennas in the frequency domain can be expressed as [23]

$$
\begin{aligned}
& \mathbf{Y}_{1}=\mathbf{S E}_{1}(\tau) \mathbf{B}+\mathbf{W}_{1}, \\
& \mathbf{Y}_{2}=\mathbf{S E}_{2}(\varsigma) \mathbf{B}+\mathbf{W}_{2},
\end{aligned}
$$

where $\mathbf{B}=\left[\begin{array}{lllll}\beta_{1} & \beta_{2} & \ldots & \beta_{K}\end{array}\right], \mathbf{W}_{1}=\left[\begin{array}{llll}\mathbf{w}_{1}^{(1)} & \mathbf{w}_{2}^{(1)} & \ldots & \mathbf{w}_{K}^{(1)}\end{array}\right]$, and $\mathbf{W}_{2}=\left[\begin{array}{llll}\mathbf{w}_{1}^{(2)} & \mathbf{w}_{2}^{(2)} & \ldots & \mathbf{w}_{K}^{(2)}\end{array}\right] \cdot \mathbf{E}_{1}(\tau)$ and $\mathbf{E}_{2}(\varsigma)$ are the delay matrices of the two antennas, respectively, which can be expressed as

$$
\begin{gathered}
\mathbf{E}_{1}(\tau)=\left[\begin{array}{cccc}
\mathbf{e}_{1}\left(\tau_{1}\right) & \mathbf{e}_{1}\left(\tau_{2}\right) & \ldots & \mathbf{e}_{1}\left(\tau_{L}\right)
\end{array}\right] \\
=\left[\begin{array}{cccc}
1 & 1 & \cdots & 1 \\
e^{-j \Delta \omega \tau_{1}} & e^{-j \Delta \omega \tau_{2}} & \ldots & e^{-j \Delta \omega \tau_{L}} \\
\vdots & \vdots & \ddots & \vdots \\
e^{-j(N-1) \Delta \omega \tau_{1}} & e^{-j(N-1) \Delta \omega \tau_{2}} & \cdots & e^{-j(N-1) \Delta \omega \tau_{L}}
\end{array}\right], \\
=\left[\begin{array}{cccc}
\mathbf{E}_{2}(\varsigma)=\left[\begin{array}{cccc}
\mathbf{e}_{2}\left(\varsigma_{1}\right) & \mathbf{e}_{2}\left(\varsigma_{2}\right) & \ldots & \mathbf{e}_{2}\left(\varsigma_{L}\right)
\end{array}\right] \\
1 & \cdots & 1 \\
1 & e^{-j \Delta \omega \varsigma_{1}} & \cdots & e^{-j \Delta \omega \varsigma_{L}} \\
\vdots & \vdots & \ddots & \vdots \\
e^{-j(N-1) \Delta \omega \varsigma_{1}} & e^{-j(N-1) \Delta \omega \varsigma_{2}} & \cdots & e^{-j(N-1) \Delta \omega \varsigma_{L}}
\end{array}\right]
\end{gathered}
$$

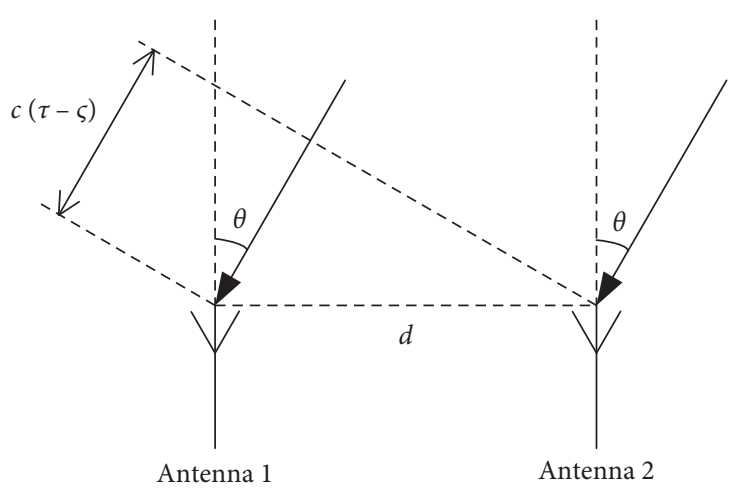

Figure 1: Array model.

The estimation of the two channel impulse responses in the frequency domain can be achieved by

$$
\begin{aligned}
& \widehat{H}_{1}=\frac{\mathbf{Y}_{1}}{\mathbf{S}}=\mathbf{E}_{1}(\tau) \mathbf{B}+\mathbf{V}_{1}, \\
& \widehat{H}_{2}=\frac{\mathbf{Y}_{2}}{\mathbf{S}}=\mathbf{E}_{2}(\varsigma) \mathbf{B}+\mathbf{V}_{2},
\end{aligned}
$$

where $\mathbf{V}_{1}=\left(\mathbf{W}_{1} / \mathbf{S}\right)$ and $\mathbf{V}_{2}=\left(\mathbf{W}_{2} / \mathbf{S}\right)$. In the next section, we propose a joint TOA and DOA estimation algorithm utilizing the channel impulse response $\widehat{H}_{1}$ and $\widehat{H}_{2}$.

\section{Proposed Algorithm}

3.1. Sample Points and Clusters Extension. The cross-correlation matrix of the channel impulse response can be constructed as

$$
\mathbf{R}_{H}=E\left\{\mathbf{H}_{1} \mathbf{H}_{2}^{H}\right\} \approx \mathbf{E}_{1}(\tau) \mathbf{R}_{B} \mathbf{E}_{2}^{H}(\varsigma),
$$

where $\mathbf{R}_{B}=E\left\{\mathbf{B} \mathbf{B}^{H}\right\} \in \mathbb{C}^{L \times L}$ is a diagonal matrix. We divide the cross-correlation matrix $\mathbf{R}_{H} \in \mathbb{C}^{N \times N}$ into two $N \times(N-$ 1) matrices, i.e.,

$$
\begin{aligned}
& \mathbf{X}_{1}=\mathbf{R}_{H}(:, 1: N-1)=\mathbf{E}_{1}(\tau) \mathbf{R}_{B} \mathbf{E}_{2 a}^{H}(\varsigma), \\
& \mathbf{X}_{2}=\mathbf{R}_{H}(:, 2: N)=\mathbf{E}_{1}(\tau) \mathbf{R}_{B} \mathbf{E}_{2 b}^{H}(\varsigma),
\end{aligned}
$$

where $\mathbf{X}_{1}$ and $\mathbf{X}_{2}$ consist of the first and the last $N-1$ columns of $\mathbf{R}_{H}$ and $\mathbf{E}_{2 a}(\varsigma)$ and $\mathbf{E}_{2 b}(\varsigma)$ consist of the first and the last $N-1$ rows of $\mathbf{E}_{2}(\varsigma)$. According to the form of the delay matrix, we have

$$
\mathbf{E}_{2 b}(\varsigma)=\mathbf{E}_{2 a}(\varsigma) \Psi(\varsigma)
$$

where $\Psi(\varsigma)$ is an $L \times L$ diagonal matrix given by

$$
\boldsymbol{\Psi}(\varsigma)=\left[\begin{array}{llll}
e^{-j \Delta \omega \varsigma_{1}} & & & \\
& e^{-j \Delta \omega \varsigma_{2}} & & \\
& & \ddots & \\
& & & e^{-j \Delta \omega \varsigma_{L}}
\end{array}\right] .
$$

According to (9) and (10), the two delay matrices of the two antennas are Vandermonde matrices and have the characteristic of conjugate symmetry, i.e., 


$$
\begin{aligned}
& \mathbf{J}_{N} \mathbf{E}_{1}^{*}(\tau)=\mathbf{E}_{1}(\tau) \widetilde{\Phi}(\tau), \\
& \mathbf{J}_{N} \mathbf{E}_{2}^{*}(\varsigma)=\mathbf{E}_{2}(\varsigma) \widetilde{\Phi}(\varsigma),
\end{aligned}
$$

where $\mathbf{J}_{N} \in \mathbb{C}^{N \times N}$ is the antidiagonal matrix, and the rotation matrices $\widetilde{\Phi}(\tau)$ and $\widetilde{\Phi}(\varsigma)$ are, respectively, given by

$$
\begin{aligned}
& \widetilde{\Phi}(\tau)=\left[\begin{array}{lll}
e^{-j \Delta \omega \tau_{1}} & & \\
& e^{-j \Delta \omega \tau_{2}} & \\
& & e^{-j \Delta \omega \tau_{L}}
\end{array}\right], \\
& \widetilde{\Phi}(\varsigma)=\left[\begin{array}{lll}
e^{-j \Delta \omega \mathcal{C}_{1}} & \\
& e^{-j \Delta \omega \mathcal{\zeta}_{2}} & \\
& & e^{-j \Delta \omega \mathcal{L}_{L}}
\end{array}\right] .
\end{aligned}
$$

By utilizing the above properties of the delay matrices, we can construct a new matrix using $\mathbf{X}_{1}$ and $\mathbf{X}_{2}$ as

$$
\mathbf{X}=\left[\begin{array}{l}
\mathbf{X}_{1}, \mathbf{J}_{N} \mathbf{X}_{2}^{*} \\
\mathbf{X}_{2}, \mathbf{J}_{N} \mathbf{X}_{1}^{*}
\end{array}\right]=\mathbf{E}_{e}(\tau, \varsigma) \mathbf{B}_{e}(\tau, \varsigma)
$$

where

$$
\begin{aligned}
& \mathbf{E}_{e}(\tau, \varsigma)=\left[\begin{array}{c}
\mathbf{E}_{1}(\tau) \\
\mathbf{E}_{1}(\tau) \boldsymbol{\Psi}^{*}(\varsigma)
\end{array}\right] \in \mathbb{C}^{2 N \times L}, \\
& \mathbf{B}_{e}(\tau, \varsigma)=\left[\mathbf{R}_{B} \mathbf{E}_{2 a}^{H}(\varsigma) \widetilde{\Phi}(\tau) \mathbf{R}_{B} \boldsymbol{\Psi}(\varsigma) \mathbf{E}_{2 a}^{T}(\varsigma)\right] \in \mathbb{C}^{L \times 2(N-1)} .
\end{aligned}
$$

Equation (19) can be seen as the equivalent channel impulse response with doubled sample points and increased number of clusters, which can improve the maximum number of detectable signals as well as the estimation performance.

3.2. Reduced-Dimension TOA Estimation. The correlation matrix of the extended observation matrix $\mathbf{X}$ can be constructed as $\mathbf{R}_{X}=\mathbf{X X}^{H}$. By applying the eigenvalue decomposition, the correlation matrix can be decomposed as

$$
\mathbf{R}_{X}=\mathbf{U}_{s} \boldsymbol{\Lambda}_{s} \mathbf{U}_{s}^{H}+\mathbf{U}_{v} \boldsymbol{\Lambda}_{v} \mathbf{U}_{v}^{H},
$$

where $\mathbf{U}_{s}$ and $\mathbf{U}_{v}$ are the signal subspace and the noise subspace, respectively, and $\Lambda_{s}=\operatorname{diag}\left\{\begin{array}{llll}\lambda_{1} & \lambda_{2} & \ldots & \lambda_{L}\end{array}\right\}$ and $\Lambda_{v}=\operatorname{diag}\left\{\begin{array}{llll}\lambda_{L+1} & \lambda_{L+2} & \ldots & \lambda_{2 N}\end{array}\right\}$ are diagonal matrices consisting of the largest $L$ eigenvalues and the smallest $2 N-L$ eigenvalues of $\mathbf{R}_{X}$.

Similar to the classical MUSIC algorithm, we can construct the 2D-MUSIC spectrum as

$$
f_{2 D}(\tau, \varsigma)=\frac{1}{\mathbf{e}_{e}^{H}(\tau, \varsigma) \mathbf{U}_{v} \mathbf{U}_{v}^{H} \mathbf{e}_{e}(\tau, \varsigma)},
$$

where

$$
\mathbf{e}_{e}(\tau, \varsigma)=\left[\begin{array}{c}
\mathbf{e}_{1}(\tau) \\
\mathbf{e}_{1}(\tau) e^{j \Delta \omega \varsigma}
\end{array}\right]
$$

Apparently, (22) needs time-consuming 2D spectral search to obtain TOA estimates. Aiming to reduce the computational complexity, we split $\mathbf{e}_{e}(\tau, \varsigma)$ as

$$
\mathbf{e}_{e}(\tau, \varsigma)=\left[\begin{array}{c}
1 \\
e^{j \Delta \omega \varsigma}
\end{array}\right] \otimes \mathbf{e}_{1}(\tau)=\left(\mathbf{I}_{2} \otimes \mathbf{e}_{1}(\tau)\right) \mathbf{q}(\varsigma),
$$

where $\mathbf{q}(\varsigma)=\left[1, e^{j \Delta \omega \varsigma}\right]^{T}$. Substituting (24) into (23), the spectrum function can be rewritten as

$$
f_{2 D}(\tau, \varsigma)=\frac{1}{\mathbf{q}^{H}(\varsigma) \mathbf{F}(\tau) \mathbf{q}(\varsigma)},
$$

where $\mathbf{F}(\tau)=\left(\mathbf{I}_{2} \otimes e_{1}(\tau)\right)^{H} \mathbf{U}_{v} \mathbf{U}_{v}^{H}\left(\mathbf{I}_{2} \otimes \mathbf{e}_{1}(\tau)\right)$ and the vector $\mathbf{q}(\varsigma)$ satisfies $\mathbf{u}^{H} \mathbf{q}(\varsigma)=1$ with $\mathbf{u}=[1,0]^{T}$. Equation (25) can be regarded as the following optimization problem:

$$
\begin{aligned}
& \min _{\tau, \varsigma} \mathbf{q}^{H}(\varsigma) \mathbf{F}(\tau) \mathbf{q}(\varsigma) \\
& \text { s.t. } \mathbf{u}^{H} \mathbf{q}(\varsigma)=1 .
\end{aligned}
$$

According to (26), we can construct the cost function as

$$
L(\tau, \varsigma)=\mathbf{q}^{H}(\varsigma) \mathbf{F}(\tau) \mathbf{q}(\varsigma)-\rho\left(\mathbf{u}^{H} \mathbf{q}(\varsigma)-1\right),
$$

where $\rho$ is a constant value. In order to get the extremum, we can construct the partial derivation of $L(\tau, \varsigma)$ with respect to $\mathbf{q}(\varsigma)$, i.e.,

$$
\frac{\partial L(\tau, \varsigma)}{\partial \mathbf{q}(\varsigma)}=2 \mathbf{F}(\tau) \mathbf{q}(\varsigma)+\rho \mathbf{u}=0
$$

Thus, we have $\mathbf{q}(\varsigma)=\mu \mathbf{F}^{-1}(\tau) \mathbf{u}$ with $\mu=-0.5 \rho$. Considering $\mathbf{u}^{H} \mathbf{q}(\varsigma)=1$, the constant $\mu$ can be further expressed as

$$
\mu=\frac{1}{\mathbf{u}^{H} \mathbf{F}^{-1}(\tau) \mathbf{u}} .
$$

Therefore, the vector $\mathbf{q}(\varsigma)$ can be further transformed into

$$
\mathbf{q}(\varsigma)=\frac{\mathbf{F}^{-1}(\tau) \mathbf{u}}{\mathbf{u}^{H} \mathbf{F}^{-1}(\tau) \mathbf{u}}
$$

Substituting (30) into (26), then the TOA estimation result of the first antenna is given by

$$
\widehat{\tau}=\underset{\tau}{\arg \min } \frac{1}{\mathbf{u}^{H} \mathbf{F}^{-1}(\tau) \mathbf{u}}=\underset{\tau}{\arg \max } \mathbf{u}^{H} \mathbf{F}^{-1}(\tau) \mathbf{u},
$$

which means we can get the TOA estimation of the first antenna by a $1 \mathrm{D}$ spectral search with the spectral function

$$
f(\tau)=\mathbf{u}^{H}\left(\left(\mathbf{I}_{2} \otimes \mathbf{e}_{1}(\tau)\right)^{H} \mathbf{U}_{v} \mathbf{U}_{v}^{H}\left(\mathbf{I}_{2} \otimes \mathbf{e}_{1}(\tau)\right)\right)^{-1} \mathbf{u} .
$$

Similarly, in order to obtain the TOA estimates of the second antenna, we can exchange the order of $\mathbf{H}_{1}$ and $\mathbf{H}_{2}$ when constructing the cross-correlation matrix, i.e.,

$$
\mathbf{R}_{H}=E\left\{\mathbf{H}_{2} \mathbf{H}_{1}^{H}\right\} \approx \mathbf{E}_{2}(\varsigma) \mathbf{R}_{B} \mathbf{E}_{1}^{H}(\tau) .
$$


Then, by following the same procedures described above, the $1 \mathrm{D}$ spectral function with respect to the TOA of the second antenna is given by

$$
f(\varsigma)=\mathbf{u}^{H}\left(\left(\mathbf{I}_{2} \otimes \mathbf{e}_{2}(\varsigma)\right)^{H} \mathbf{U}_{v} \mathbf{U}_{v}^{H}\left(\mathbf{I}_{2} \otimes \mathbf{e}_{2}(\varsigma)\right)\right)^{-1} \mathbf{u} .
$$

After obtaining the TOA estimates of both antennas, we can estimate the DOA of the impinging signals by using the TOA estimates as well as the geometric information, i.e.,

$$
\widehat{\theta}_{l}=\arcsin \left(\frac{c\left(\widehat{\tau}_{l}-\widehat{\varsigma}_{l}\right)}{d}\right), \quad l=1,2, \ldots, L .
$$

The main steps of the proposed algorithm are summarized as follows:

(1) Construct the cross-correlation matrix $\mathbf{R}_{H}$ according to (12).

(2) Divide $\mathbf{R}_{H}$ into $\mathbf{X}_{1}$ and $\mathbf{X}_{2}$ according to (13) and (14), and construct the extended observation matrix $\mathbf{X}$ as (19).

(3) Compute the correlation matrix of $\mathbf{X}$, and perform the eigenvalue decomposition to obtain the noise subspace $\mathbf{U}_{v}$.

(4) Construct the spectral function according to (32) and perform the $1 \mathrm{D}$ spectral search to obtain the TOA estimates $\widehat{\tau}$.

(5) Reconstruct $\mathbf{R}_{H}$ using (33) and repeat Step 2 and Step 3. The TOA estimates $\widehat{\varsigma}$ can be obtained by performing 1D spectral search on (34).

(6) Calculate the DOA estimates according to (35).

\section{Performance Analysis}

4.1. Complexity. According to Section 3, when estimating the TOA of the first antenna $\tau$, constructing $\mathbf{R}_{H}$ needs $O\left\{N^{2} K\right\}$, computing $\mathbf{R}_{X}$ needs $O\left\{8 N^{2}(N-1)\right\}$, and performing eigenvalue decomposition on it requires $O\left\{8 N^{3}\right\}$. Constructing the spectral function and conducting $1 \mathrm{D}$ spectral search needs $O\left\{4 N^{2}(2 N-L)+8 n_{s}\left(N^{2}+N+1\right)\right\}$, where $n_{s}$ denotes the search times. Therefore, the total complexity of the proposed algorithm is $O\left\{2 N^{2} K+48 N^{3}-8 N^{2}(L+2)+16 n_{s}\left(N^{2}+N+1\right)\right\}$. If we perform $2 \mathrm{D}$ spectral search on equation (22), then the complexity is increased to $O\left\{N^{2} K+24 N^{3}-\right.$ $\left.4 N^{2}(L+2)+2 n_{s}^{2} N(2 N+1)\right\}$. The computational complexity of the proposed algorithm and some other algorithms are concluded in Table 1. Besides, the complexity comparison versus the number of frequency sample points of different algorithms is depicted in Figure 2, where $K=100$, $L=3$, and $n_{s}=100$. As shown in Figure 2, the complexity of the proposed algorithm is remarkably reduced compared with the algorithms with $2 \mathrm{D}$ spectral search and is close to the complexity of the 2D-PM.

4.2. Algorithm Advantages. We conclude the advantages of the proposed algorithm as follows:

(1) The proposed algorithm reduces the computational complexity by transforming the $2 \mathrm{D}$ spectral search to twice 1D spectral search.

(2) The proposed algorithm doubles the equivalent frequency sample points and increases the equivalent number of clusters, which can improve the maximum number of detectable signals as well as the estimation accuracy.

(3) The proposed algorithm can obtain higher estimation accuracy compared with some existed algorithms.

\section{Simulation Results}

In this section, we perform large number of Monte Carlo trials to examine the effectiveness as well as the superiority of the proposed algorithm. The root mean square error (RMSE) of the trials is used to measure the estimation accuracy, which is given by

$$
\begin{aligned}
\mathrm{RMSE}_{\mathrm{TOA}} & =\frac{1}{L} \sum_{l=1}^{L} \sqrt{\frac{1}{Q} \sum_{q=1}^{Q}\left(\left(\tau_{l}-\widehat{\tau}_{l, q}\right)^{2}+\left(\varsigma_{l}-\widehat{\varsigma}_{l, q}\right)^{2}\right)}, \\
\mathrm{RMSE}_{\mathrm{DOA}} & =\frac{1}{L} \sum_{l=1}^{L} \sqrt{\frac{1}{Q} \sum_{q=1}^{Q}\left(\theta_{l}-\widehat{\theta}_{l, q}\right)^{2}},
\end{aligned}
$$

where $Q$ is the number of Monte Carlo trials and $\widehat{\tau}_{l, q}, \widehat{\varsigma}_{l, q}$, and $\widehat{\theta}_{l, q}$ are the estimation result of $\tau_{l}, \varsigma_{l}$, and $\theta_{l}$ in the $q$ th trial, respectively. In the following simulations, we set $Q=500$, $d=0.09 \mathrm{~m}$, and $L=3$ with $\tau=[0.2 \mathrm{~ns}, 0.3 \mathrm{~ns}, 0.4 \mathrm{~ns}]$.

Figures 3 and 4 show the TOA estimation results of the proposed algorithm, where each point represents a trial, $K=100, N=64$, and the signal-to-noise ratio (SNR) is $-10 \mathrm{~dB}$ and $10 \mathrm{~dB}$, respectively. As shown in the two figures, the proposed algorithm can successfully obtain the paired TOA estimation results and the estimation accuracy is improved with higher SNR.

Figures 5 and 6, respectively, compare the TOA and DOA estimation accuracy with different number of clusters, where $N=64$. The simulation results show that the estimation accuracy of both TOA and DOA improves with increased number of clusters.

Figures 7 and 8, respectively, depict the TOA and the DOA estimation performance comparison versus SNR of different algorithms, where $K=100$ and $N=64$. It is illustrated clearly that the proposed algorithm can achieve 
TABLE 1: Complexity comparison of different algorithms.

\begin{tabular}{lc}
\hline Algorithm & Complexity \\
\hline Proposed & $O\left\{2 N^{2} K+48 N^{3}-8 N^{2}(L+2)+16 n_{s}\left(N^{2}+N+1\right)\right\}$ \\
2D proposed & $O\left\{N^{2} K+24 N^{3}-4 N^{2}(L+2)+2 n_{s}^{2} N(2 N+1)\right\}$ \\
Successive PM [24] & $(6 K+4 L+1) N^{2}+3 L^{2} N+\left(3 L^{3}+4 L+2\right)(N-1)+3 L^{3}$ \\
& $+2(2 N-L)^{3}+4 N(2 N-L)^{2}+2 n_{s}(2 N+1)(2 N-L)$ \\
2D-PM [14] & $(6 K+4 L+1) N^{2}+2 L^{2} N+L^{3}+2(2 N-L)^{3}$ \\
Matrix pencil [19] & $+4 N(2 N-L)^{2}+n_{s}^{2}(2 N+1)(2 N-L)$ \\
& $(2 K+1) N^{2}+\left(6 K L^{2}+2 K\right) N-2 K L^{3}$ \\
\hline
\end{tabular}

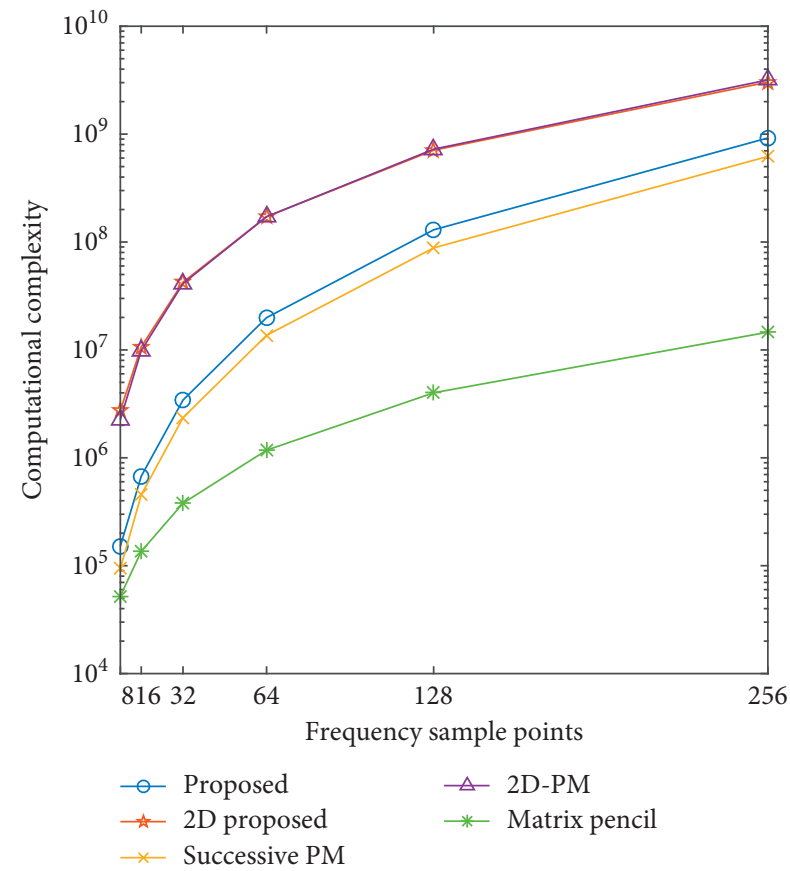

Figure 2: Complexity comparison of different algorithms, where $K=100, L=3$, and $n_{s}=100$.

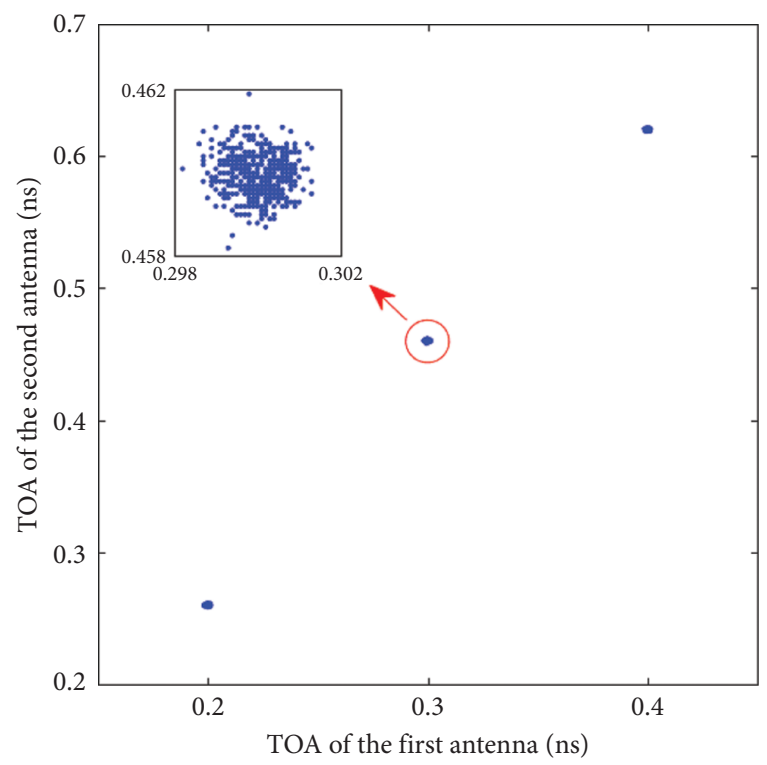

FIGURE 3: Estimation results of the proposed algorithm, where $\mathrm{SNR}=-10 \mathrm{~dB}, K=100$, and $N=64$.

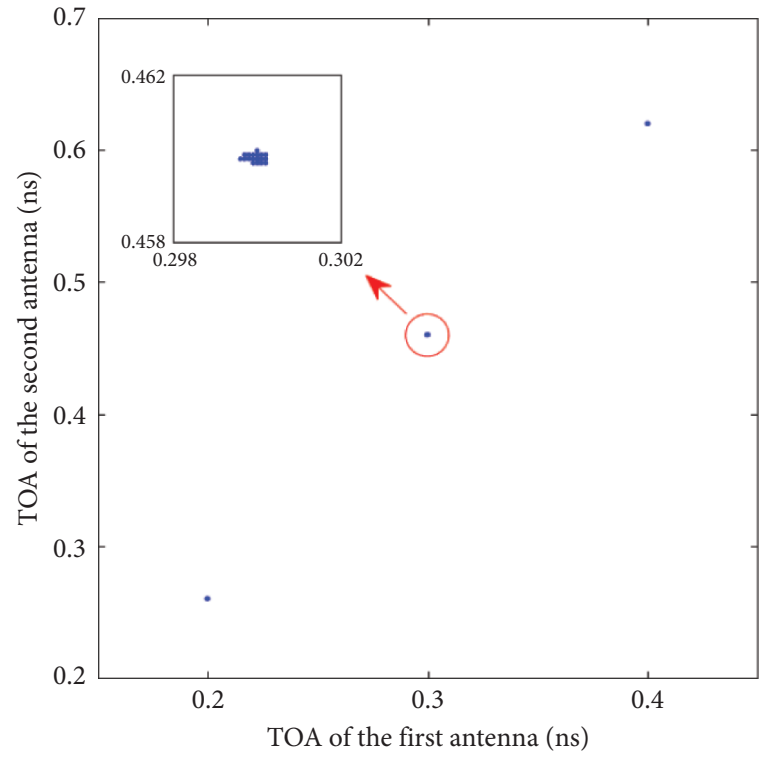

FIGURE 4: Estimation results of the proposed algorithm, where $\mathrm{SNR}=10 \mathrm{~dB}, K=100$, and $N=64$.

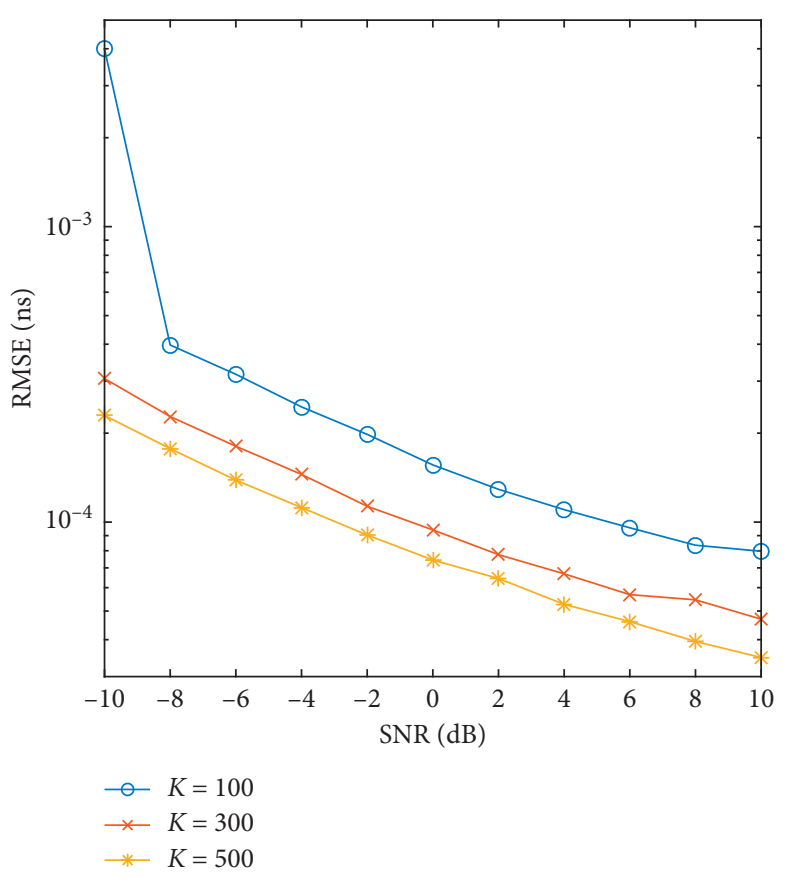

FIGURE 5: TOA estimation performance of the proposed algorithm in different number of clusters, where $N=64$. 


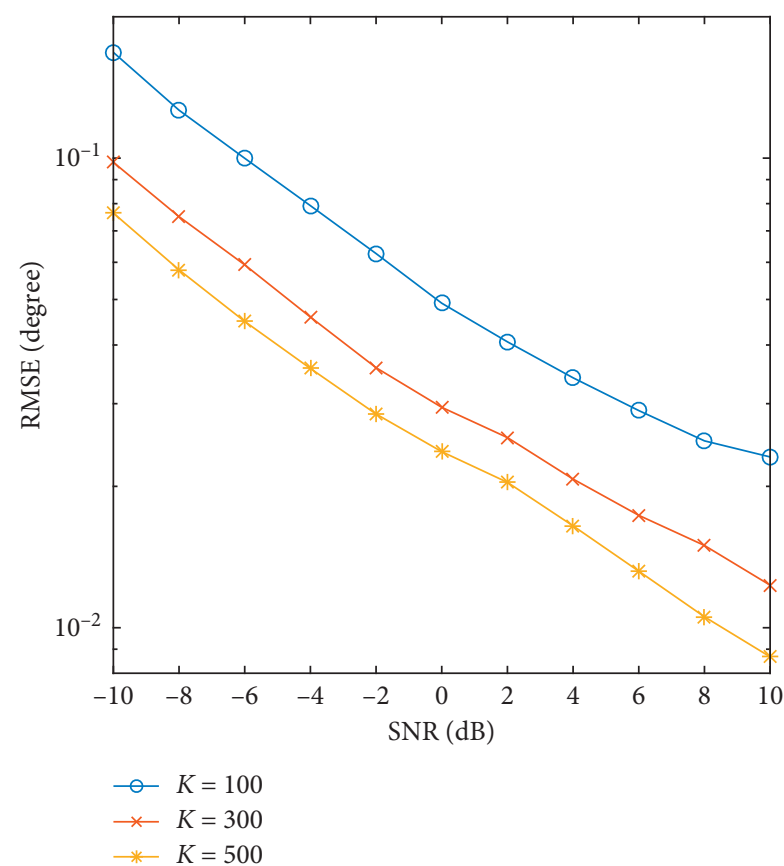

FIGURE 6: DOA estimation performance of the proposed algorithm in different number of clusters, where $N=64$.

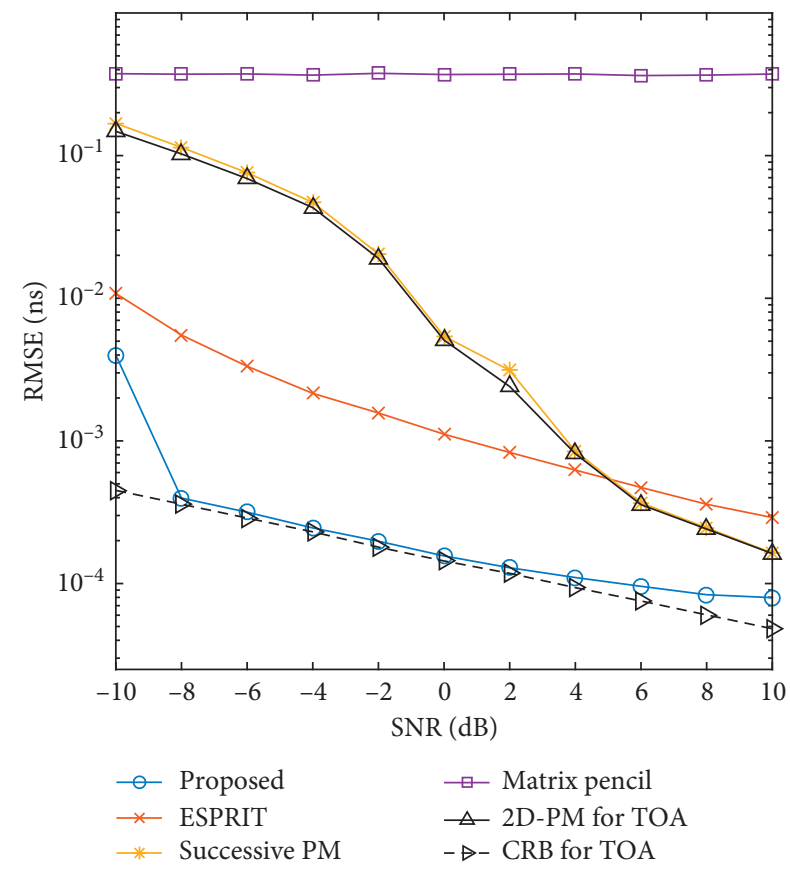

FIGURE 7: TOA estimation performance comparison of different algorithms, where $K=100$ and $N=64$.

better estimation performance in both TOA and DOA domains compared with the ESPRIT algorithm [16], the successive PM [24], the matrix pencil algorithm [19], and the 2D-PM [14].

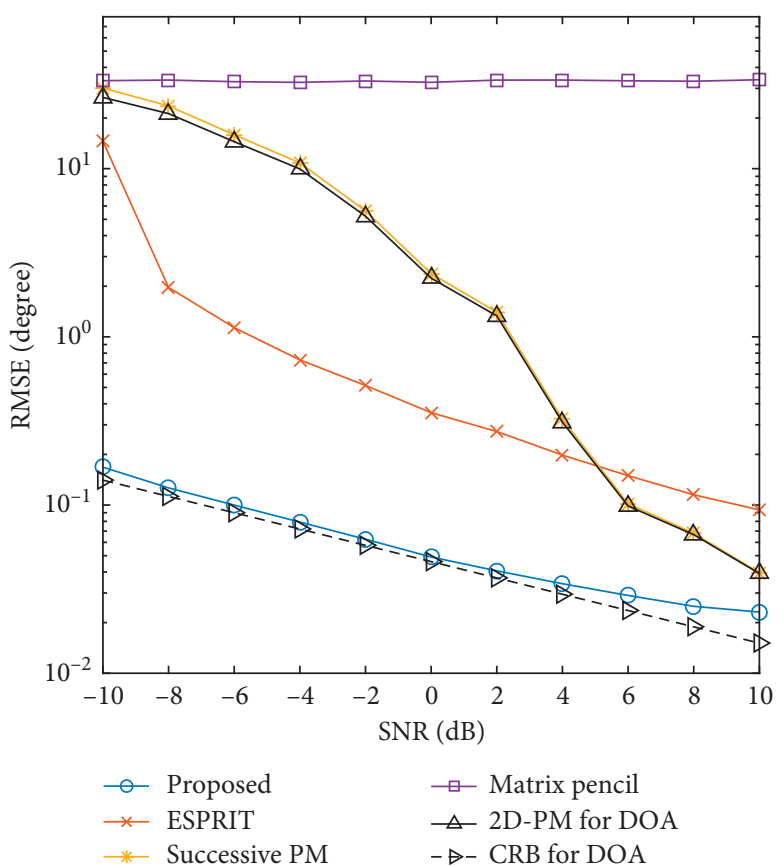

FIGURE 8: DOA estimation performance comparison of different algorithms, where $K=100$ and $N=64$.

\section{Conclusion}

In this paper, we investigate the problem of joint TOA and DOA estimation in UWB systems and propose a computationally efficient algorithm with doubled frequency sample points and extended number of clusters. The proposed algorithm utilizes the conjugate symmetry characteristic of the delay matrices to extend the sample points as well as the number of clusters and then transforms the 2D spectral search into twice 1D search in order to reduce the computational complexity. Simulations testify that the proposed algorithm can obtain high estimation accuracy and large number of identifiable signals with low complexity.

\section{Data Availability}

The data used to support the findings of this study are included within the article.

\section{Conflicts of Interest}

The authors declare that they have no conflicts of interest.

\section{Acknowledgments}

This work was supported by Application of Beidou and Ultra Wideband High Precision Positioning Technology in Ubiquitous Power Internet of Things (51230N19000T). 


\section{References}

[1] H. Soganci and S. Gezici, "Accurate positioning in ultrawideband systems," IEEE Wireless Communications, vol. 18, no. 2, pp. 19-27, 2011.

[2] J. Rovnakova and D. Kocur, "Short range tracking of moving persons by UWB sensor network," in Proceedings of the 2011 European Radar Conference (EuRAD), pp. 321-324, Manchester, UK, May 2011.

[3] T. Sakamoto and T. Sato, "A target tracking method with a single antenna using time-reversal UWB radar imaging in a multi-path environment," in Proceedings of the 2010 IEEE International Geoscience and Remote Sensing Symposium (IGARSS), pp. 3319-3322, Honolulu, HI, USA, June 2010.

[4] Y. Luo and C. L. Law, "Indoor positioning using UWB-IR signals in the presence of dense multipath with path overlapping," IEEE Transactions on Wireless Communications, vol. 11, no. 10, pp. 3734-3743.

[5] M. R. Mahfouz and M. J. Kuhn, "UWB channel measurements and modeling for positioning and communications systems in the operating room," in Proceedings of the 2011 IEEE Topical Conference on Biomedical Wireless Technologies, Networks, and Sensing Systems (BioWireleSS), pp. 47-50, Phoenix, AZ, USA, November 2011.

[6] L. Joon-Yong and R. A. Scholtz, "Ranging in a dense multipath environment using an UWB radio link," IEEE Journal on Selected Areas in Communications, vol. 20, no. 9, pp. 1677-1683, 2002.

[7] A. F. Molisch, K. Balakrishnan, C. Chong, et al., IEEE 802.15.4a Channel Model-Final Report, http://www.ieee802. org/15/pub/TG4a.html.

[8] S. Wu, Q. Zhang, R. Fan et al., "Match-filtering based TOA estimation for IR-UWB ranging systems," in Proceedings of the 2008 International Wireless Communications and Mobile Computing Conference (IWCMA '08), pp. 1099-1105, Crete, Greece, August 2008.

[9] I. Guvenc, Z. Sahinoglu, and P. V. Orlik, "TOA estimation for IR-UWB systems with different transceiver types," IEEE Transactions on Microwave Theory and Techniques, vol. 54, no. 4, pp. 1876-1886, 2006.

[10] A. A. D'Amico, U. Mengali, and L. Taponecco, "Energy-based TOA estimation," IEEE Transactions on Wireless Communications, vol. 7, no. 3, pp. 838-847, 2008.

[11] I. Guvenc and Z. Sahinoglu, "Threshold selection for UWB TOA estimation based on kurtosis analysis," IEEE Communications Letters, vol. 9, no. 12, pp. 1025-1027, 2005.

[12] K. Pahlavan, X. Li, and J. P. Makela, "Indoor geolocation science and technology," IEEE Communications Magazine, Vo.vol. 40, no. 2, pp. 112-118, 2002.

[13] X. Li and K. Pahlavan, "Super-resolution TOA estimation with diversity for indoor geolocation," IEEE Transactions on Wireless Communications, vol. 3, no. 1, pp. 224-234, 2004.

[14] J. Hong, F. Cao, and D. Rui, "Propagator method-based TOA estimation for UWB indoor environment in the presence of correlated fading amplitudes," in Proceedings of the 2008 4th IEEE International Conference on Circuits and Systems for Communications (ICCSC), pp. 535-538, Shanghai, China, May 2008.

[15] F. Zhao, W. Yao, C. C. Logothetis et al., "Comparison of super-resolution algorithms for TOA estimation in indoor IEEE 802.11 wireless LANs," in Proceedings of the 2006 International Conference on Wireless Communications, Networking and Mobile Computing (WiCOM), pp. 1-5, Wuhan, China, October 2006.
[16] H. Saarnisaari, "TLS-ESPRIT in a time delay estimation," in Proceedings of the IEEE 47th Vehicular Technology Conference, pp. 1619-1623, Phoenix, Arizona, USA, May 1997.

[17] D. G. Oh, S. H. Jang, J. W. Chong et al., "High resolution TOA estimation algorithm uing IEEE 802.15.4a CSS signal," in Proceedings of the 2008 4th International Conference on Wireless Communications, Networking and Mobile Computing (WiCOM'08), pp. 1-3, Dalian, China, October 2008.

[18] D. Rui, Z. Qian, and H. Jiang, "TOA estimation for IR-UWB systems using matrix pencil," World Congress on Computer Science and Information Engineering, vol. 1, pp. 461-464, 2009.

[19] D. Rui, Z. Qian, and W. Xue, "Joint TOA and DOA estimation of IR-UWB system based on matrix pencil," International Forum on Information Technology and Applications (IFITA'09), vol. 1, pp. 544-547, 2009.

[20] M. Navarro and M. Najar, "Frequency domain joint TOA and DOA estimation in IR-UWB," IEEE Transactions on Wireless Communications, vol. 10, no. 10, pp. 1-11, 2011.

[21] F. Wen, J. Shi, and Z. Zhang, "Closed-form estimation algorithm for EMVS-MIMO radar with arbitrary sensor geometry," Signal Processing, vol. 186, pp. 1-9, 2021.

[22] H. Bo and N. C. Beaulieum, "Accurate performance evaluation of time-hopping and direct-sequence UWB systems in multi-user interference," IEEE Transactions on Communications, vol. 53, no. 6, pp. 1053-1062, 2005.

[23] A. A. M. Saleh and R. A. Valenzuela, "A statistical model for indoor multipath propagation," IEEE Journal on Selected Areas in Communications, vol. 5, no. 2, pp. 128-137, 1987.

[24] F. Wang, X. Zhang, C. Wang et al., "Joint estimation of TOA and DOA if IR-UWB system using a successive propagator method," International Journal of Electronics, vol. 102, no. 10, pp. 1765-1788, 2015. 